\title{
Standards for Web Applications on Mobïle: current state and roadmap
}

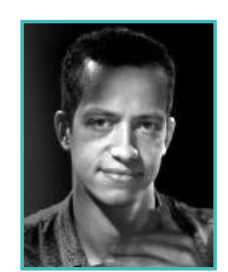

Dominique Hazaël-Massieux supervises the development and standardization of Web technologies that are most relevant to mobile devices in W3C, and is in charge of the W3C groups that are developing APIs to access more device capabilities from the Web (camera, addressbook, etc) and to enable peer-to-peer audio-video communication in Web browsers (WebRTC).

$\mathrm{He}$ also regularly puts in practice these technologies and guidelines as a developer of a number of sites and applications.
Web technologies have become powerful enough that they are used to build full-featured applications; this has been true for many years in the desktop and laptop computer realm, but is increasingly so on mobile devices as well.

This tutorial will introduce the various technologies developed in W3C that increase the capabilities of Web applications, and how they apply more specifically to the mobile context. 